\title{
Dimensions of Situatedness for Digital Public Displays
}

\author{
Rui José, ${ }^{1}$ Nuno Otero, ${ }^{2}$ and Jorge C. S. Cardoso ${ }^{3}$ \\ ${ }^{1}$ Algoritmi Centre, University of Minho, 4800-058 Guimarães, Portugal \\ ${ }^{2}$ Department of Media Technology, Faculty of Technology, Linnoeus University, 35195 Växjö, Sweden \\ ${ }^{3}$ CITAR/School of Arts, Portuguese Catholic University, 4169-005 Porto, Portugal \\ Correspondence should be addressed to Rui José; rui@dsi.uminho.pt
}

Received 30 May 2014; Revised 27 November 2014; Accepted 28 November 2014; Published 22 December 2014

Academic Editor: Marco Mamei

Copyright (C) 2014 Rui José et al. This is an open access article distributed under the Creative Commons Attribution License, which permits unrestricted use, distribution, and reproduction in any medium, provided the original work is properly cited.

\begin{abstract}
Public displays are often strongly situated signs deeply embedded in their physical, social, and cultural setting. Understanding how the display is coupled with on-going situations, its level of situatedness, provides a key element for the interpretation of the displays themselves but is also an element for the interpretation of place, its situated practices, and its social context. Most digital displays, however, do not achieve the same sense of situatedness that seems so natural in their nondigital counterparts. This paper investigates people's perception of situatedness when considering the connection between public displays and their context. We have collected over 300 photos of displays and conducted a set of analysis tasks involving focus groups and structured interviews with 15 participants. The contribution is a consolidated list of situatedness dimensions that should provide a valuable resource for reasoning about situatedness in digital displays and informing the design and development of display systems.
\end{abstract}

\section{Introduction}

Public displays, of all kinds, are one of the most ubiquitous elements of our visual culture. From signage and road signs to billboards, posters or notice boards, as we look around in our environment we are constantly facing a very broad range of public displays with multiple form factors serving very diverse purposes. Most of these displays are strongly situated; in other words, they are deeply embedded in their specific physical, social, and cultural setting and they are already an integral part of established practices. This close coupling with its particular situation or circumstances is inherently a twoway relationship in which display and environment become strongly interdependent. The meaning of the situated display can be better interpreted within the context in which it is inscribed, but at the same time displays become a central element for the interpretation of space, its situated practices, and its social context or place. Situated displays can foster social coordination and collaboration, allowing different resources to be interpreted and used effectively in social action. Furthermore, they help to create a shared sense of place by transmitting the identity and purpose of a space, inviting people to action, setting behavior expectations, or supporting navigation within contextual boundaries. As summarized by O'Hara et al., "... they inform us about places, amenities, and events of interest and reflect the activities of others [...]. They act as important cultural reference points in the construction of shared meanings, beliefs, desires and the memories of groups and communities." [1]. Situatedness is also important in allowing us to move within the overwhelming amount of information in our environment while fluidly focusing at any time on the information that is most relevant to our current activity.

The motivation for this work originates from the observation that most digital displays in public settings are very far from achieving the same level of situatedness that seems so natural in their nondigital counterparts. Huang et al. [2] point out how the vast majority of large displays in public areas are designed without proper consideration for the ways in which the display setting, that is, space, the people around, and the local activities, may affect the perceived relevance of those displays, thus yielding suboptimal situations, lower utility, and less attention. Müller et al. [3] report on how audience expectations towards what is presented on digital 
public displays, that is, mostly boring advertisements and trivial content, correlate with their attention towards these displays and produce an effect of display-blindness in which there is a tendency to ignore the displays. These findings suggest that in digital displays the complexity brought by technology may be somehow obliterating elements of design that are key enablers for situatedness.

Our long-term goal is to identify the range of design elements that may improve the integration between digital displays and their physical and social context. We have conducted a study based on an extensive collection of over 300 photos of public displays that served as the basis for interviews with 15 participants about their perspectives on the situatedness elements represented in those displays. At the end, we have consolidated the results emerging from the interviews into a set of situatedness dimensions and we have analyzed their implications for digital situated displays. The contribution of this work is twofold: (1) we identify and characterize 6 dimensions built from people's perception of situatedness in public displays, more specifically: location, spatial, activity, community, perceived ownership, and place identity; (2) we frame the integration of these dimensions in the design of digital display systems by analyzing how they could address the specificities of the digital medium. This contribution should provide a new framework for reasoning about situatedness support in these display systems, help other researchers and practitioners to approach the topic from a more explicit perspective, and promote the exploration of a broader range of design possibilities for digital situated displays.

\section{Related Work}

In regard to situated digital displays in particular, situatedness has mainly been approached either from a design perspective or by exploring the sensing capabilities of digital displays.

In the design perspective, a particular setting is studied to inform the design of a specific display concept. The process involves considering the local practices, how the place is perceived by people, and, possibly, the types of practices that it is meant to support. Designers are then able to make informed decisions that are sensitive to these issues and create a display experience to explicitly convey a specific sense of place. For example, in the Shannon Portal Installation by Ciolfi et al. [4], a series of field studies have led to an interactive installation designed to allow people to share their travelling experience based on their photos. The authors emphasize that when designing for public environments it is essential to understand the place in its entirety, including the system's physical and material qualities. Graham et al. [5] describe field work done at a residential community care facility that investigated the setting and discussed technology designs with care workers. The result is described as providing a broader understanding of the care workers' needs and informing on how and where public display technology could be deployed.

The design of situated displays for specific local communities has been extensively explored [6], with particular incidence in work environments as a means to disseminate information or provide awareness about group activities [7, 8]. O'Hara et al. [9] describe how a situated display appliance showing basic room reservation information outside the meeting room could become an important resource for social action around the use of that space. Their observations highlight how the information on the displays was regarded as a resource for action rather than an absolute rule and also how local knowledge about activities in the office was implicitly used to interpret what the information of the displays really meant. Odom et al. [10] describe how the situated visualization of water consumption in dormitory communities can be used as a tool for promoting sustainable behaviors. The situated and communal nature of the display is described as a key element in peer-pressure and community awareness. The Whereabouts Clock [11] that displays the approximate location of family members and the HomeNote [12] that displays SMS messages and notes sent by family members are also examples of situated displays providing awareness about the location or activities within a family. They serve as tools for coordination between the family members and implicitly reflect their daily routines.

Together, these systems demonstrate how multiple concepts of situatedness can be explored with digital displays and also how a properly conducted design approach is able to incorporate into the displays a broad sensibility to physical, social, and aesthetic concerns. However, the effort needed to conduct such design processes is considerable and the results are not easy to generalize to other displays because the entire process was meant to address the specificities of one particular installation. Our purpose is to identify at least a few generic elements that could enable digital public displays to systematically align their behavior to their usage circumstances. While it can be argued that a specific design will always be able to fit the specific situation for which it was created in a way that a generic approach will never fit, we believe that the elements/dimensions we intend to uncover can guide designers towards the creation of many more situated displays, even if in a more limited way, than what would ever be possible to achieve with dedicated designs.

The idea of creating displays that are able to dynamically sense and react to the changing environment around them is something that has been explored in numerous ways. Farnham et al. [13] describe how a sense of community and place attachment can be promoted by displaying media associated with the profiles of people present in a café. McCarthy et al. refer to these displays as proactive displays and study how presence, detected either through RFID tags or Bluetooth, is used as a trigger for displaying profiles of nearby people, in an attempt to promote occasional encounters [14]. The Hello.Wall [15] is also a proactive display that detects the presence of specialized personal devices, called viewports, and presents ambient information emerging from the patterns of presence and activity. MobiTip [16] explores the visualization of Bluetooth based interactions (or tips) advertised by nearby devices. The BluScreen [17] uses Bluetooth detection to avoid showing advertisements to users more than once. Content 
that has already been shown when a particular Bluetooth device was present is avoided if that device is present again, thus reducing the likelihood of the same content being shown again to the same person. Müller and Krüger [18] propose a model for learning the spatiotemporal behavior of users and then enable a situated display to estimate profiles that could be used for presentation on the display of the information that is most interesting to users. The Peddler Interaction Framework [19] incorporates continuous proxemic measures such as distance and orientation, attention states, such as digression and loss of interest, and the passerby's interaction history. The goal is to adjust the display's behavior in such a way that maximizes the ability to attract attention, maintain interest, create desire, and lead customers to action. Instant Places [20] enables people to express their content preferences in the form of pins that are recognized when the user checks in to a display using a mobile client. The displays will then preferably select the content sources associated with those pins. The Funsquare application [21] presents trivia information in a way that reflects the current context around the display.

This ability to sense and react to their environment is an obvious path for situated displays and is very much aligned with the field of context awareness, albeit in this case focusing on the shared context of place. Like context awareness in general, this approach faces a clear gap between what can be sensed and perceived by the system and what is perceived by people as the situation around the display. Even though it seems highly intuitive to think that certain contextual variables can affect what is the most appropriate content to be presented in a particular situation, it is far from trivial to actually specify in the system behavior the subtle influences of those elements.

In general, the interplay between technology and its placement has long been recognized as central to many types of systems. The work by Dourish [22] explores how embodiment, described as a physical presence in the world and a social embedding in a web of practices, can play a key role in the design of interactive systems. Paay and Kjeldskov [23] propose a conceptual framework for situated social interactions in public and illustrate how their framework informed the design of mobile context-aware applications. Leahu et al. advocate that context-aware systems should promote a tight integration between sensing and action rather decomposing them as separate parts of the same process and focusing on abstract representations of context [24].

While previous work has mainly focused on interaction itself and specific context-sensitive behaviors, we explore a systematic approach to the notion of situatedness where our main concern is the multiple ways in which this notion can be captured, become readily available as a design resource, and decisively frame the design of the system. More specifically, we consider that there may be many dimensions involved in the notion of situatedness and that explicit support for each of them should offer a rich set of design alternatives from which specific situated display concepts will emerge. The situated behavior of any display would thus be the outcome of various forms of adaptation across multiple situatedness dimensions.

\section{Research Design}

To a certain extent, any display can be described as situated, in the sense that it is embedded with some particular cultural or language background. Rather than trying to define the boundaries for what may be considered a situated display or classifying types of situated displays, we have chosen to focus on the identification of dimensions of situatedness, that is, particular ways in which a display design may be linked to the specific situation in which it is inscribed. The goal is to identify the elements that may lead to some sort of design toolbox from which multiple alternative strategies can be used to create displays that are more tightly integrated with their situation.

To study these dimensions, we followed a research methodology comprising 3 phases: exploration, interviews, and consolidation.

3.1. Phase 1: Exploration. Our work started with an exploratory study within our team to formulate an initial understanding of the problem domain and generate an overview of the range of situatedness elements that may be found on public displays. We carried out a fieldwork activity in which we collectively gathered images from various types of public displays. This process went on for a period of about three months, during which over 300 photos were collected, about a third of which representing digital displays. We leveraged on the richness and diversity offered by opportunistic encounters with many types of displays in real usage to approach a crowdsourcing model in which we were able to expand the scope of this study far beyond what we in our own team could have envisioned as possible display concepts.

We then organized a focus group with 7 participants (2 females, 5 males, aged 22-45 years) selected among the people working at our research lab, but not directly involved in this research. The group analyzed the photos and discussed the possible concepts of situatedness embedded in each display. For each photo, participants were asked to indicate one or more elements that connected that display to its setting. These elements were then registered in a board and reused with subsequent photos whenever appropriate. If there was not an obvious match among the already existing elements, then a new element was created. At the end, the resulting elements were analyzed and we conducted a simple consolidation process that generated 8 categories of similar concepts. The goal of this process was to increase the representativeness of the set of display photos that were going to be used in the subsequent interview phase. The 8 categories identified at this stage were never again used as part of this research.

The 8 categories identified in this exploration phase allowed us to create a selection of 30 display photos that we believe are representative of the many ways in which displays can be situated. We started by selecting 3 photos from each of those categories. We then added 6 more photos in which there was a strong combination of more than one category. We considered this to be manageable in size, considering our next goal of conducting interviews, and yet representative of the rich diversity of display types. Some of those photos are shown in Figure 1. 

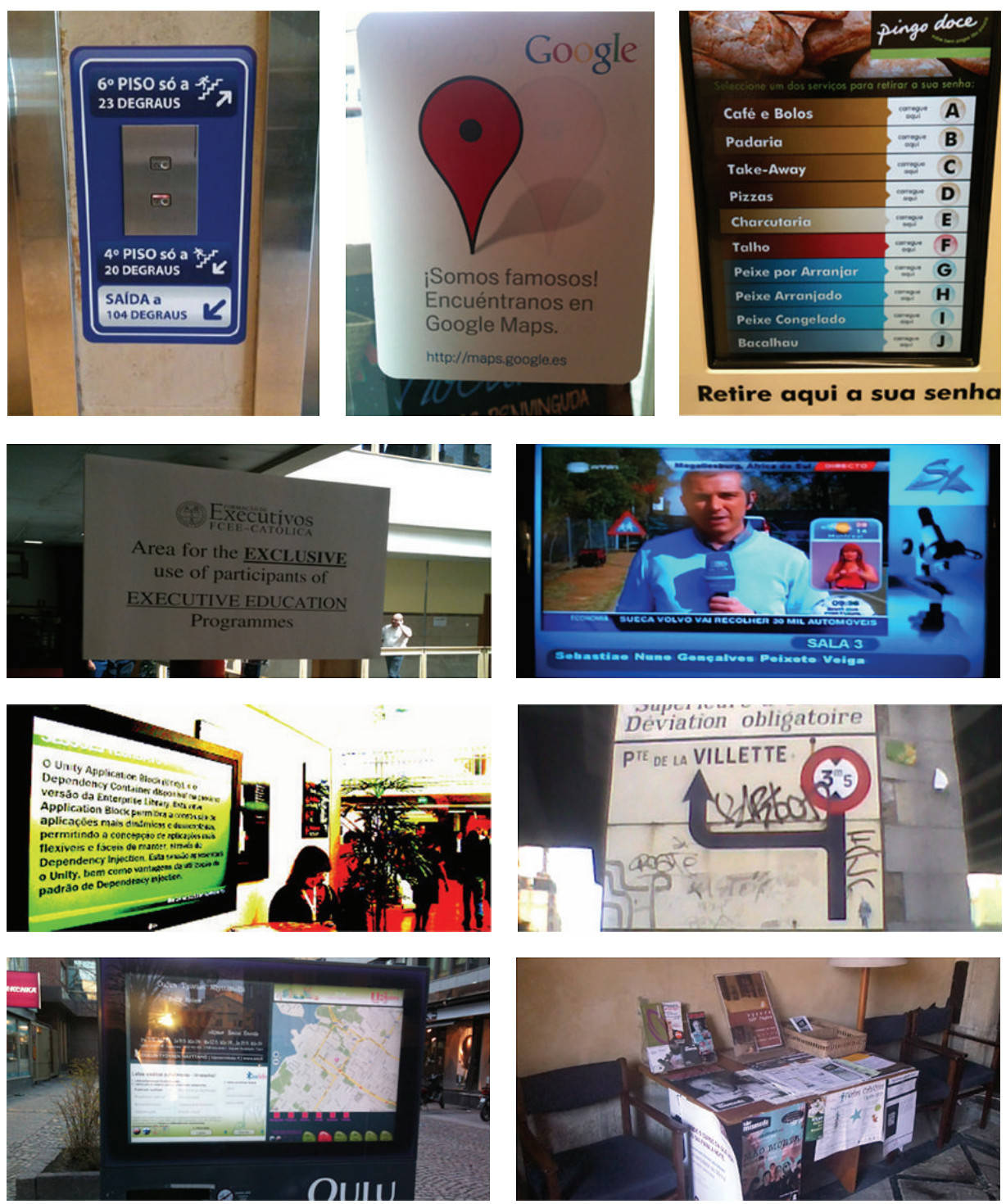

FIGURE 1: Examples of photos used in the interviews.

3.2. Phase 2: Interviews. In the second phase, we conducted semistructured interviews to uncover individual perceptions about the elements that support the link between public displays and their situation. We recruited 15 participants aged between 20 and 45 . The participants were rewarded with a $5 €$ voucher on a local bookshop for their collaboration in the study. To promote some diversity in the participants, they were recruited at 2 different locations: the university and a local council social center. The interviews also took place at the locations where participants had been recruited and they were centered on the analysis of the 30 display photos selected in the exploration phase.

Each participant was interviewed about 4 of those photos, and for each of them they answered questions in a semistructured interview format aiming at eliciting elements that people regarded as important to connect the display with its setting. There were no mentions whatsoever to the general concept of situatedness or to the initial categories identified in phase 1. Instead, people were shown the photos and asked to evoke possible scenarios associated with that display. More specifically, they were asked (a) to talk about the display's utility, (b) why it was attractive, (c) what were the main activities around the display, (d) to invent a short story about why that display had been put there, (e) to create another story about a possible usage scenario related with that display, and (f) what arguments could be used to convince the place owner to install such display. Finally, at the end of the scenario characterization, we also probed people about what elements they felt could have facilitated the interpretation of the display, if they were in its real setting, rather than just seeing it as a photo.

The transcripts of the interviews were then analyzed by two researchers who had not been involved in the first phase. While being a type of analysis that is always sensible to some 
subjectivity, because of the qualitative nature of the data generated by the interviews, we followed established best practices to minimize bias and subjectivity. The analysis was based on an open coding data analysis approach. The researchers annotated any indication that could be interpreted as a reference to the situation in which the display was integrated. From the coding of the data, a set of concepts emerged related to displays' situatedness, which was accompanied by descriptions to facilitate the understanding when reanalyzing the findings. The whole process had an iterative nature, as new codes were continually compared and possibly merged with existing codes and the existing descriptions were refined. The result was a list with 29 elementary concepts/codes associated with situatedness.

3.3. Phase 3: Consolidation. In the consolidation phase, we tried to characterize the major themes emerging from the 29 situatedness elements identified in the previous phase. This process was based on the generation of an affinity diagram. The process began with the creation of paper labels corresponding to the code descriptions. These labels were then distributed and discussed within the research team to create a shared understanding of their meaning and the participants' answers behind them. We then moved forward with the creation of the affinity diagram with all the researchers moving labels to form clusters of similar concepts and conflicting decisions being discussed by the entire team. The result was the identification of 6 main themes corresponding to the key dimensions of situatedness for public displays, more specifically, location, spatial association, activity, community, perceived ownership, and place identity. We then went back to the original data associated with each of the dimensions to create the descriptions that can be found in the next section. To better illustrate the connection between a particular dimension and the specific ways in which it can be perceived by people, we included representative transcription from the interviews.

\section{Dimensions of Situatedness}

This section presents the different dimensions elicited/created following the empirical work described in the previous sections. Each dimension is described and examples of the comments made during the interviews that helped us frame each concept are also provided.

4.1. Location of the Display. The location dimension considers the forms of situatedness that result directly from the location of the display. This may include concepts such as a map of the neighborhood, the local weather forecast, or the broken ATM that informs about alternative ATMs nearby. Regarding a display showing a map of a city, a participant has observed how he expected to find information about the nearby attractions: "[...] it's useful city information, such as 'you are here', with key points of interest in the city." The notion of proximity is frequently implicit, even though the scope of what should be seen as proximate is very fuzzy and strongly dependent on the content itself. Participants indicated their expectation that a photo display on a photo shop would be showing photos mainly from the city or region, but also from the shop itself: "[...] the photographs show what goes on inside [...]". This demonstrates how location proximity can implicitly be seen as the basis for many other forms of situatedness, such as perceived ownership or community. However, we are only considering the situatedness concepts that result directly from physical proximity in the same sense as in location-based services. For content situated in regard to location, the physical position of the display is a defining element of that content, and it should be clear in what way that content would change if the display was to be moved to some other location.

4.2. Spatial Association. The spatial dimension considers the spatial arrangement of the display with respect to its environment. In these cases, fine-grained placement or the orientation of the display can be critical for the interpretation of its meaning. Situatedness emerges directly from their position with relation to a particular object or small region or the specific orientation of the display within its surrounding space. This includes the most traditional forms of signage and navigation assistance. In signage, spatial placement is done in a way that allows the reader to make the association between the display and the object or place it refers to. The display is describing, identifying, or calling attention to a place or object to which it is attached in a way that makes the association clear. Participants have clearly identified this spatial attachment and referred to those displays as informing them about specific properties of space. Interestingly, similar references were made in relation to displays creating explicit awareness about digital services, locally available but otherwise invisible, such as signs for Wi-Fi ("[...] I looked for a panel that informed me about internet availability [...]") and Bluetooth hotspots and a Google Maps logo displayed in a restaurant door as a sign of web presence.

In navigation scenarios, the information presented is aligned with the specific position and orientation of the display. This includes any displays pointing the direction to another destination, and also maps that are physically aligned with the area being represented. In these specific cases, the object to which they are attached is normally not very relevant, but their exact positioning and orientation is crucial. Even a slight change in position or orientation may be enough to change the way the display is going to be interpreted: "[...] there is more than one direction, and the panel tells us we should turn right."

To a certain extent, spatial association also involves location, and there are several borderline cases between both forms of situatedness, for example, a local map aligned with the cardinal points. They are, however, very distinct in the sense that location is bound by scope, while spatial association is bound by physical placement; that is, there is a much stronger coupling between the display placement and the information being shown. For content situated in regard to spatial association, the placement of the display within its immediate surrounding space can be so crucial to the interpretation of that content that any minor change in position or orientation may be enough to affect or even subvert the interpretation of that content. 
4.3. Activity. The activity dimension considers the way in which a display reflects on-going activities taking place in the display setting. At the most basic level, this may involve raising awareness about those activities. One participant, assuming a display to be about a local exhibition, commented that "It would be important to guide people that are passingby, so that they know what is happening inside the exhibition hall." Dynamic displays, however, have the additional ability to represent the status of activities. The natural dynamics of the activity generates a continuous flow of state changes and events that is somehow relevant for most people in that place. The display can then become a focal point for coordination and a decision-making tool as, for example, when deciding which movie to watch at a cinema or which car park to go to depending on current availability displayed on a street display. A display reflecting the status of locally on-going activities can thus become strongly situated considering the potential dynamics of the information being presented and also the immediate relevance of the information to people engaged in those activities. This is especially the case with strongly programmed places, in which there is a clearly dominant activity surrounding the display that largely sets information needs. Typical examples may include departures/arrivals displays on a train station or queuing information on a waiting area.

Assumptions about surrounding activities are a strong source of situatedness because they can align the content of the display with immediate needs of people seeing the display. For example, a display beginning with the message "Tired of waiting? Why not ..." was pointed out as being clearly designed for a queuing area and will be read by people who identify themselves with that situation. This notion of opportunity has also been associated with the expectation that people watching the display will have specific time and attention spans that can be considered when designing the display. Participants have often mentioned how the need to wait at a particular location could make them more receptive to certain types of content.

For content in close connection with local activities, the function of the place becomes a defining element of that content, and it should be clear how that content would lose its meaning if the connection between the display and the predominant activity was lost. Consider, for example, how the situatedness of a scoreboard in a stadium would be broken during a music concert or if its content froze during a game and the displayed score was no longer updated.

4.4. Community. This dimension considers the ways in which the display can become a reflection of the community that shares a place in which it is inscribed. There is also the assumption that the display is somehow open to the community and that situatedness emerges from the strong potential for engagement of content that is locally generated, published, or selected. This is normally associated with community notice boards, classified ads in supermarkets, or informal leaflets displays, but additional examples may include graffiti and stickers posted on street furniture.

The concepts extracted from the interviews seem to point at two slightly different, albeit complementary, ways in which community situatedness can emerge. The first and most obvious is that situatedness emerges from the set of people that predominantly frequent the place and are therefore the potential contributors for the display. The community becomes represented in the display because some of its members are contributing or influencing content and therefore reflecting their interests on the display.

However, participants have also pointed out how certain displays were perceived as being targeted at a specific community: "this is in a central place and is there for tourists visiting the city." In some cases, participants, not feeling part of that target community, have clearly expressed their lack of identification and even their inability to understand the content: "It is showing something that must be for a specific audience, maybe students." An interesting example emerged with a leaflet display on a bookstore. The leaflets composing that display are contributions of the community, but, as observed by one of the participants, they also reflect the expectation of the leaflet distributors about the community frequenting that place. The explicit act of leaving a particular leaflet in a particular place is embedded with an implicit understanding of the social setting of the place and its predominant community. An informal leaflet display in a public place can thus become a unique representation of how a crowd of contributors interprets the appropriateness of that place for passing a particular message to a specific target community.

For content situated in regard to the community, the predominant users of a place and their interests become a defining element of the display content and it should be clear how that content would change if that community also substantially changed. Consider, for example, how a display in a conference center, showing interests and messages of conference participants would change when in the next day a different conference would be taking place.

4.5. Perceived Ownership. The perceived ownership dimension considers the ways in which the perception that a certain entity is responsible for the display is embedded in the display content. This association can be explicit, but since most displays are owned by whoever owns the location where they are placed, this link is often implicit from the display location. For example, a shopping center or a hotel have clear boundaries in which administrative and location scopes overlap and any displays located inside those boundaries will be perceived as owned by those entities.

Perceived ownership normally spills into perceived authorship. One of the displays used in the interviews was a paper sheet posted on the front window of a restaurant that simply said "we seek employees." In this case, the clear scope of the restaurant and thus the implicit ownership was crucial for the interpretation of the content of the message. As observed by one of the participants: "it's a strong connection, because it's in there that they need an employee." This also includes several types of institutional information, such as opening hours, institutional policies, contacts, and recommendations on how to conduct local activities, to which participants have often referred to as general interest information. 
In this dimension, situatedness emerges not only because the displays reflect the specific view of the display owner on what general interest might be, but also because the perception of who is the content creator is central to the meaning of the message.

Shared displays exhibiting content from many sources should be designed to preserve this form of situatedness by clearly highlighting the source or the author of each content item, rather than simply assuming that content alone is enough for the interpretation of the respective message.

4.6. Place Identity. This final dimension considers the connection between the display design and the perceived identity of the place. It entails a design process in which a more or less explicit interpretation of place becomes embedded in the way a display is experienced. This may include displays that try to contribute towards building a specific place identity or displays that are designed to reflect identity elements of the place where they are deployed.

A striking example emerging from the interviews was the case of the large commercial billboards in Times Square, New York. Participants have clearly associated their design with the prestige of the place and observed how, in such place, being there and the size of the respective display were really the most important part of the message and an essential element in the interpretation of those displays: "it gives us the idea of a big city, lots of movement, lots of stores, lots of commercial activity."

Similarly, participants have also mentioned how a digital photo display with an attractive design could increase the perception that the photo shop where it was deployed was very innovative and high-tech. Also, a display with leaflets about cultural events in a book shop was identified as contributing to the identity of the book shop as a place of culture: "it's a place where there is a book shop, it's a place of culture, it [the display] fits perfectly in the place." A wood frame display with information about a national park was also mentioned as another example of a display clearly designed for the specific setting.

Finally, commercial displays in general and all sorts of other identity creation displays, such as artistic works, may also be described as being embedded with specific views on how the place should be experienced by people or even as ways to promote particular states of mind or expectations.

\section{Implications for Digital Displays}

We will now discuss the implications of our findings and propose dimensions to the emergence of new concepts of situated digital displays. More specifically, we analyze how the dimensions that have been identified in the study may provide a framework for the integration of situatedness elements in the design of digital displays and how the specificities of the digital medium can affect the potential sources of situatedness.

The first step in this process is to acknowledge that digital displays are a new medium with its own specific properties, and therefore we cannot simply expect the processes through which situatedness becomes embedded in the displays to be exactly the same as in their nondigital counterparts.

With nondigital displays, the processes leading to situatedness are essentially driven by a broad range of wellestablished practices surrounding the creation, placement, maintenance, and appropriation of public displays. These practices are supported by a very broad and widely available set of content publication tools and display embodiments and they are also strongly embedded in implicit social behaviors, reflecting the various forms of negotiation around the design and use of public artifacts and the rich subtleties of local knowledge.

These properties of nondigital displays enable people to have a great degree of control over their use and appropriate them for a multitude of communication purposes. Digital displays, however, are still quite constrained in the range of situated practices that they can accommodate (maybe also due to the novelty of the artifact itself as referred to previously in this paper). Content is often distributed from a central point to many locations and spontaneous content publication is not conveniently supported. There are many more constraints on possible form factors and deployment placements, and above all, they lack a set of well-established tools and techniques that could enable people to appropriate them for whatever purposes they might have.

Considering that situatedness in digital displays cannot be grounded on the exact same assumptions and processes as in nondigital displays, our present analysis cannot just be about trying to understand how to emulate in digital displays the same sources of situatedness. It should also be about trying to uncover new approaches that are aligned with the specificities of the digital medium, as the latter may call for alternative processes of situatedness that were not previously supported by nondigital displays. Digital displays can be dynamic and much more prone to being interactive or reactive to the situation around them. They can alternate between multiple content pieces and they can access frequently updated content. They can even adapt their behavior according to the changing environment around them or the interactions they receive. Consequently, digital displays can be reactive to situations rather than just being designed for situations. We believe this is crucial characteristic and it will be central for this analysis. More specifically, it means that digital displays can use this unique capability to autonomously and proactively adapt to the situations as an alternative path towards situated behavior. They can explore the ability to acquire information about their situation to behave in ways that can be perceived by people as rational, enticing, or engaging for the current situation.

The ability to react to what is happening around rather than being conceived from scratch for a specific setting, is closer to the views of situatedness that are commonly used in robotics $[25,26]$. From that perspective, situatedness characterizes an agent in which "behavior and cognitive processes first and foremost are the outcome of a close coupling between agent and environment" [27]. The intelligence of the agent is directly linked to the strength of this relation and the ability of the agent to behave in a way that looks rational according to 
the continuously changing and unpredictable circumstances of the surrounding environment.

Exhibiting this smart behavior requires a broader framework for dealing with situatedness in a more explicit way, allowing the behavior of a situated digital display to support the dynamic integration of situation elements from the various dimensions. We should note, however, that this does not necessarily mean representing or modeling situations. The intelligence of an agent should reveal itself in the ways in which it integrates well with existing practices, very much in the same sense as a home can be seen as intelligent because if it can properly support the practices of its inhabitants [28] or its displays evolve with the unique needs of each environment [29].

5.1. Towards Situated Behavior in Digital Displays. Considering these specificities of the digital medium and the properties of the various dimensions of situatedness, we will now analyze the possible strategies for integrating situated behavior into digital displays.

Location situatedness is one dimension in which the adaptive capabilities of public displays can be a major advantage. Despite some differences in the underlying assumptions, location adaptation is very similar to the concept of locationbased services as commonly used in the context of mobile applications. This is thus an area in which there is the possibility to benefit from an already existing and extensive body of knowledge and also from the increasing availability of location information. The only technical requirement would be a procedure to allow the display to expose location information to the applications that generate location-based content. With this information, it should be simple to have centralized content sources that could generate situated content on each location in which they are used. It should also be simple to stick a display anywhere and have it immediately presenting content associated with that location, such as the local weather, local news, or tweets in the surroundings. This is thus a dimension that should be easy to support by taking advantage of the adaptive capabilities of digital public displays.

Spatial association is a situatedness dimension that is strongly affected by the differences between the digital and nondigital media. In nondigital displays, physical placement normally plays the key role as a source of situatedness. Displays may exist in large numbers in an environment and their embodiment and specific placement can be carefully selected to provide the best match with the display purpose. Therefore, they can be designed, positioned, and oriented in a way that maximizes the spatial situatedness of the respective content. Digital displays, on the contrary, face many more limitations on the number and type of deployments. They are considerably more costly to deploy, not just because of the display devices themselves, but also because of their associated infrastructures. Even when considering the dropping costs of new displays technologies, there are still technical and safety limitations that may impose additional constraints on form factors and their possible locations. As a consequence, there will normally be fewer digital displays per location and their placement will be largely affected by considerations about deployment constraints and overall visibility. They will rarely be used to present a very specific piece of content to an occasional audience or designed to go unnoticed until someone needs them. Instead, they will be placed with the expectation that they will serve a sufficiently broad range of content to a sufficiently large audience to justify their cost. The need to serve multicontent and the fact that placement is almost always selected independently of content mean that it will be harder to align physical placement and embodiment with content. However, we can expect a trend towards cheaper displays that can be attached to meaningful locations and in which spatial association will be the main driver for situatedness. These spatially situated digital displays include examples such as digital menu boards, room reservation systems [9], or systems like the Hermes office door displays [30] in which people can leave messages that are associated with the occupant of the office to which the display is attached. There is also scope for adaptive procedures that generate content based on where the display is positioned. The generation of content that is spatially situated requires much more than just the location. It should also describe the placement of the display in close relation with the surrounding physical environment. Depending on the specific types of information, this may imply fine-grained position, orientation, viewing angles, and possibly a model of the surrounding environment [31]. This way the display would be able to show information embedded with knowledge about the spatial arrangement of the display, such as referring to nearby objects, direction arrows, or "you are here type of maps" for indoors. This is similar in concept to the way some augmented reality systems for mobile devices work, for example, Layar, and thus a dimension in which it should be possible to benefit from an already existing body of knowledge when creating adaptation mechanisms.

The representation of on-going activities is already a common source of situatedness for digital displays. The dynamic nature of the activity makes it particularly suited to be represented by digital displays. The main challenge here regards the ability to enlarge the set of activities that can be represented. Nowadays, this is mostly limited to wellstructured processes for which there is already an existing information system in place, such as departures information or queues. With the increasingly available information about real-world events and the immediacy of locative social media, situated displays may increasingly become an alternative way to understand at a social level what has been or will be happening in the places we go to. More research is needed, however, on how to map the currently predominant activity to the local displays.

Digital displays have a very strong potential to represent the community in which they are inscribed. Their potential for rich interaction and context sensing may offer unique opportunities for generating user-centric content and dynamically adapt to their surrounding social environment. However, this implies fostering engagement and sharing control with the potential audience, both of which are still major issues for digital displays. Generating the appropriate level of participation is a crucial challenge for interactive 
displays, as they can only maintain their situational relevance if they receive regular contributions from the people around the display. However, enticing people to participate has revealed to be a recurrent problem, as reported in several studies $[32,33]$ that suggest that people have difficulties in identifying their contributions or feel uncomfortable with the consequent public exposure. The other major challenge is to approach the level of flexible and socially mediated control that is inherent to most nondigital displays. While procedures for shared control in public displays have already been explored, specifying who can publish what, for how long, or under what circumstances, these models are still too rigid to the type of spontaneous participation that could be associated with situated displays. They fail to consider the complex and multilayered set of factors that may inhibit or promote the shared use of public display space [34]. In scenarios in which the group of potential participants is a restricted community, this is not a major problem and previous work on community-oriented displays, where user profiles can easily be associated with members of that community, has often followed this approach $[15,35,36]$. However, if the goal is to engage participation from potentially anyone coming to the display, the need to create a profile or user account in each display system may endanger the whole notion of spontaneous and open participation. Moderation is also a common alternative, but it does not scale to multiple displays and may undermine the sense of immediacy that is so central to situated interaction. Overcoming these issues requires new administrative models that facilitate multiple collaboration patterns between stakeholders and new publication practices that people may expect to find when facing a new public display.

The implications of the digital medium for perceived ownership are mainly about the increased potential for integrating and combining content from multiple sources. As we move towards more open models of control of the displays, there may be content from multiple sources and therefore this source of situatedness may become more blurred. As a design consideration, we should explicitly handle and represent content sources as a way to improve the relevance of the content. Tools for content creation and publication should explicitly acknowledge the importance of this information, which should also be clearer in the displayed content.

Identity of place is clearly the dimension where a design approach can be stronger and therefore the range of automated adaptation can be smaller. A connection between display and setting is forged through a design process that may consider a broad range of sensitivities associated with social expectations and place experience to conceive a particular display experience that conveys a strong sense of place identity. Still, not every setting needs to be studied from scratch as an isolated reality. While each café is unique in its community and context, there are certainly many common elements shared with other cafes and a broad range of generic designs that may help setting a general perspective on what might be relevant for this type of place. This should enable third parties to distribute to a potentially large display community content that is conceived specifically for particular types of place.

\section{Conclusion}

Despite the increasing body of work on public digital displays, we still need a structured framework on how to approach situatedness for digital displays, especially from a system support perspective. Clearly, we cannot just make a direct translation of the same approaches that have so successfully driven situatedness in nondigital displays. Instead, we need to develop a new set of practices and tools that may enable the various dimensions of situatedness in digital displays. In this work, we have created a more explicit understanding of the various dimensions of situatedness in public displays and discussed how they can be approached while considering the specificities of the digital medium.

Even though some of these dimensions may be found together more often than others, any of the dimensions in this list may exist on its own and we believe their possible overlap to be minimal. Still, they should not be seen as classifying displays according to one type of situatedness. We acknowledge that the full set of elements that compose the situatedness of any display can be very complex and subjective, and therefore it would not make any sense to try to reduce such reality to just one of its many dimensions. In other words, the dimensions considered are not mutually exclusive.

Also, we recognize that at a higher level of abstraction, a dimension like place identity could always be interpreted as encompassing all the others, and, admittedly, this dimension worked for us as a general umbrella for all the many and subtler forms of situatedness that we were not able to isolate as part of this study. We should note, however, that the goal of this work was not to make a contribution towards the general understanding of the complex social and physical layers that compose situatedness. Therefore, and even though we think that refining this dimension further and extracting more specific dimensions may be seen as future work, we believe to have already captured those dimensions that are more meaningful as resources for informing the design of systems support for situated displays. The set of dimensions that have been identified and the analysis of their integration in digital displays should provide an immediately valuable resource for the creation of new types of digital displays that can be perceived by people as exhibiting situated behavior.

Finally, the set of dimensions identified in this study should also be a contribution to foster communication between practitioners. The design and development of digital displays needs the active collaboration of different disciplines, as it is the case for most digital technologies that target wide audiences. This type of conceptual frameworks may facilitate the sharing and discussion of ideas and help to bridge the gap between design and development. Similar lines of argumentation can be found in the work by Cockton [37] when considering the importance of facilitating the exploration of the connections between different elements of the design space (high level concepts, user experience issues, and the actual features of the system) and improving the dialogue between distinct practitioners or by Rogers [38] when referring to the need of creating a lingua franca for HCI. We believe our current effort towards the definition of 
a set of dimensions regarding situatedness to be a step in the direction of providing a framework to facilitate this sort of collaboration within the scope of digital situated displays.

\section{Conflict of Interests}

The authors declare that there is no conflict of interests regarding the publication of this paper.

\section{References}

[1] K. O’Hara, M. Perry, E. Churchill, and D. Russell, "Introduction to public and situated displays," in Public and Situated Displays: Social and Interactional Aspects of Shared Display Technologies, K. O’Hara, Ed., Kluwer Academic Publishers, 2003.

[2] E. M. Huang, A. Koster, and J. Borchers, "Overcoming assumptions and uncovering practices: when does the public really look at public displays?" in Proceedings of the 6th International Conference on Pervasive Computing (Pervasive'08), pp. 228-243, Springer, Berlin, Germany, 2008.

[3] J. Müller, D. Wilmsmann, J. Exeler et al., "Display blindness: the effect of expectations on attention towards digital signage," in Pervasive Computing, pp. 1-8, Springer, Berlin, Germany, 2009.

[4] L. Ciolfi, M. Fernström, L. J. Bannon et al., "The Shannon portal installation: interaction design for public places," Computer, vol. 40, no. 7, pp. 64-71, 2007.

[5] C. Graham, K. Cheverst, M. Rouncefield, and C. Kray, "Going more public: situated display design in a care setting through co-realisation," in Proceedings of the Conference on Designing for User Experience (DUX '05), p. 18, 2005.

[6] N. Taylor, M. Rouncefield, K. Cheverst, and S. Izadi, "Encouraging community spirit with situated displays," in Proceedings of the AISB Symposium on Persuasive Technology, pp. 39-42, 2008.

[7] S. Greenberg and M. Rounding, The Notification Collage, ACM Press, New York, NY, USA, 2001.

[8] J. McCarthy, T. Costa, and E. Liongosari, "UniCast, OutCast \& GroupCast: an exploration of new interaction paradigms for ubiquitous, peripheral displays," in Proceedings of the Workshop on Distributed and Disappearing User Interfaces in Ubiquitous Computing at the SIGCHI Conference on Human Factors in Computer Systems (CHI '01), 2001.

[9] K. O'Hara, M. Perry, and S. Lewis, "Situated web signs and the ordering of social action," in Public and Situated Displays, K. O’Hara, E. Perry, E. Churchill, and D. M. Russel, Eds., pp. 105140, Kluwer Academic Publishers, Dordrecht, The Netherlands, 2003.

[10] W. Odom, J. Pierce, and D. Roedl, "Social incentive \& ecovisualization displays: toward persuading greater change in dormitory communities," in Proceedings of the Workshop on Public and Situated Displays to Support Communities (OZCHI '08), 2008.

[11] A. Sellen, R. Eardley, S. Izadi, and R. Harper, "The whereabouts clock: early testing of a situated awareness device," in Proceedings of the Extended Abstracts on Human Factors in Computing Systems (CHI EA '06), pp. 1307-1312, April 2006.

[12] A. Sellen, R. Harper, R. Eardley et al., "HomeNote: supporting situated messaging in the home," in Proceedings of the 20th Anniversary ACM Conference on Computer Supported Cooperative Work (CSCW '06), pp. 383-392, ACM, November 2006.
[13] S. D. Farnham, J. F. Mccarthy, Y. Patel et al., "Measuring the impact of third place attachment on the adoption of a placebased community technology," in Proceedings of the SIGCHI Conference on Human Factors in Computing Systems (CHI '09), pp. 2153-2156, 2009.

[14] J. F. McCarthy, D. H. Nguyen, A. M. Rashid, and S. Soroczak, "Proactive displays \& the experience UbiComp project," ACM SIGGROUP Bulletin, vol. 23, no. 3, pp. 38-41, 2003.

[15] T. Prante, C. Röcker, N. Streitz et al., "Hello.Wall-beyond ambient displays," in Video Track and Adjunct: Proceedings of the 5th Internnational Conference on Ubiquitous Computing (UBICOMP '03), pp. 387-409, Kluwer Academic Publishers, 2003.

[16] Å. Rudström, M. Svensson, M. Svensson, R. Cöster, and K. Höö, "MobiTip: using bluetooth as a mediator of social context," in Proceedings of the Ubiquitous Computing: 6th International Conference (UbiComp '04), 2004.

[17] M. Sharifi, T. Payne, and E. David, "Public display advertising based on bluetooth device presence," in Proceedings of the Mobile Interaction with the Real World Workshop (MIRW '06), Espoo, Finland, 2006.

[18] J. Müller and A. Krüger, "Towards situated public displays as multicast systems," in Proceedings of the Workshop on Ubiquitous User Modeling (UbiqUM '06), Riva del Garda, Italy, August 2006.

[19] M. Wang, S. Boring, and S. Greenberg, "Proxemic peddler: a public advertising display that captures and preserves the attention of a passerby," in Proceedings of the International Symposium on Pervasive Displays (PerDis '12), pp. 3-9, June 2012.

[20] R. Jose, H. Pinto, B. Silva, and A. Melro, "Pins and posters: paradigms for content publication on situated displays," IEEE Computer Graphics and Applications, vol. 33, no. 2, pp. 64-72, 2013.

[21] N. Memarovic, I. Elhart, and M. Langheinrich, "FunSquare: first experiences with autopoiesic content," in Proceedings of the 10th International Conference on Mobile and Ubiquitous Multimedia (MUM '11), pp. 175-184, ACM Press, December 2011.

[22] P. Dourish, "Where the Action Is", 2001, http://www.dourish .com/embodied/.

[23] J. Paay and J. Kjeldskov, "Understanding situated social interactions: a case study of public places in the city," Computer Supported Cooperative Work, vol. 17, no. 2-3, pp. 275-290, 2008.

[24] L. Leahu, P. Sengers, and M. Mateas, "Interactionist AI and the promise of ubicomp, or, how to put your box in the world without putting the world in your box," in Proceedings of the 10th International Conference on Ubiquitous Computing (UbiComp '08), pp. 134-143, ACM, 2008.

[25] R. Pfeifer and C. Scheier, Understanding Intelligence, MIT Press, 1999.

[26] R. A. Brooks, "Intelligence without representation," Artificial Intelligence, vol. 47, no. 1-3, pp. 139-159, 1991.

[27] J. Lindblom and T. Ziemke, "Social situatedness: Vygotsky and beyond," in Knowledge Creation Diffusion Utilization, pp. 71-78, Lund University Cognitive Studies, Lund, Sweden, 2002.

[28] A. S. Taylor, R. Harper, L. Swan, S. Izadi, A. Sellen, and M. Perry, "Homes that make us smart," Personal and Ubiquitous Computing, vol. 11, no. 5, pp. 383-393, 2007.

[29] A. Crabtree, T. Hemmings, and T. Rodden, "The social construction of displays: coordinate displays and ecologically distributed networks," in Public and Situated Displays: Social and Interactional Aspects of Shared Display Technologies, p. 422, 2003. 
[30] K. Cheverst, A. Dix, D. Fitton et al., "Exploring bluetooth based mobile phone interaction with the hermes photo display," in Proceedings of the 7th International Conference on Human Computer Interaction with Mobile Devices and Services (MobileHCI '05), pp. 47-54, ACM Press, September 2005.

[31] C. Kray, K. Cheverst, M. Harrison, F. Hamhoum, and J. Wagner, "Towards a location model for indoor navigation support through public displays and mobile devices," in Proceedings of the Mobile Interaction with the Real World Conference (MIRW '08), pp. 83-92, 2008.

[32] H. Brignull and Y. Rogers, "Enticing people to interact with large public displays in public spaces," in Proceedings of the International Conference on Human-Computer Interaction (INTERACT '03), pp. 17-24, IOS Press, 2003.

[33] E. M. Huang and E. D. Mynatt, "Shared displays for small communities: optimizing for privacy and relevance," in Proceedings of the Workshop Public, Community and Situated Displays: Computer Supported Cooperative Work (CSCW '02), 2002.

[34] F. Alt, N. Memarovic, I. Elhart, D. Bial, and A. Schmidt, "Designing shared public display networks: implications from today's paper-based notice areas," in Proceedings of the 9th International Conference on Pervasive Computing (Pervasive '11), pp. 258-275, 2011.

[35] E. F. Churchill, L. Nelson, L. Denoue, P. Murphy, J. I. Helfman, and J. Helfma, "The plasma poster network: social hypermedia on public display," in Public and Situated Displays: Social and Interactional Aspects of Shared Display Technologies, K. O'Hara, Ed., pp. 233-260, Kluwer Academic Publishers, London, UK, 2003.

[36] J. F. McCarthy, T. J. Costa, and E. S. Liongosari, "Unicast, outcast \& groupcast: three steps toward ubiquitous, peripheral displays," in Proceedings of the $3 r$ International Conference on Ubiquitous Computing (Ubicomp '01), vol. 2201 of Lecture Notes in Computer Science, pp. 332-345, Springer, 2001.

[37] G. Cockton, "Designing worth-connecting preferred means to desired ends," Interactions, vol. 15, no. 4, pp. 54-57, 2008.

[38] Y. Rogers, "New theoretical approaches for human-computer interaction," Annual Review of Information Science and Technology, vol. 38, no. 1, pp. 87-143, 2004. 

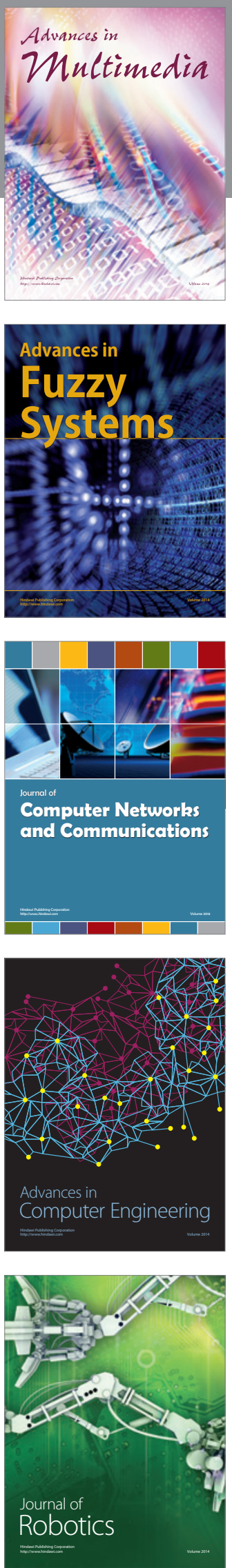

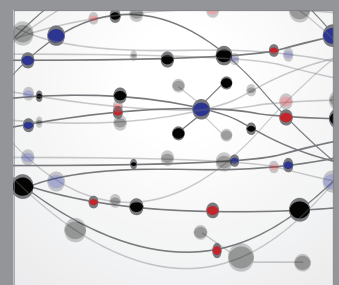

The Scientific World Journal
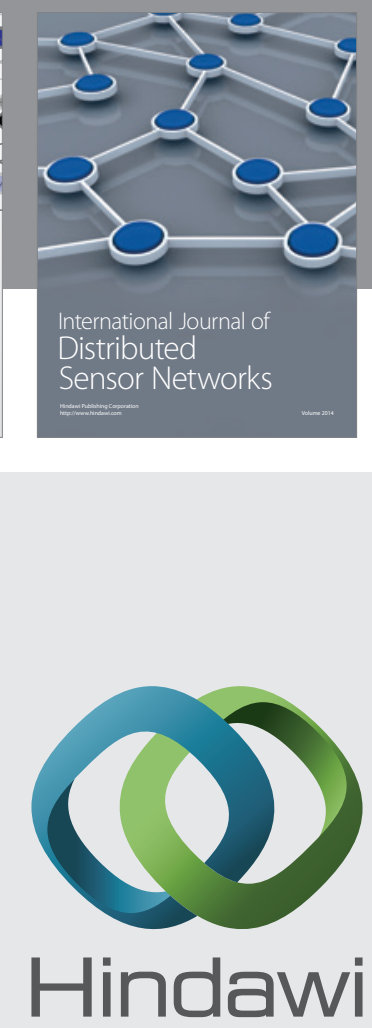

Submit your manuscripts at

http://www.hindawi.com
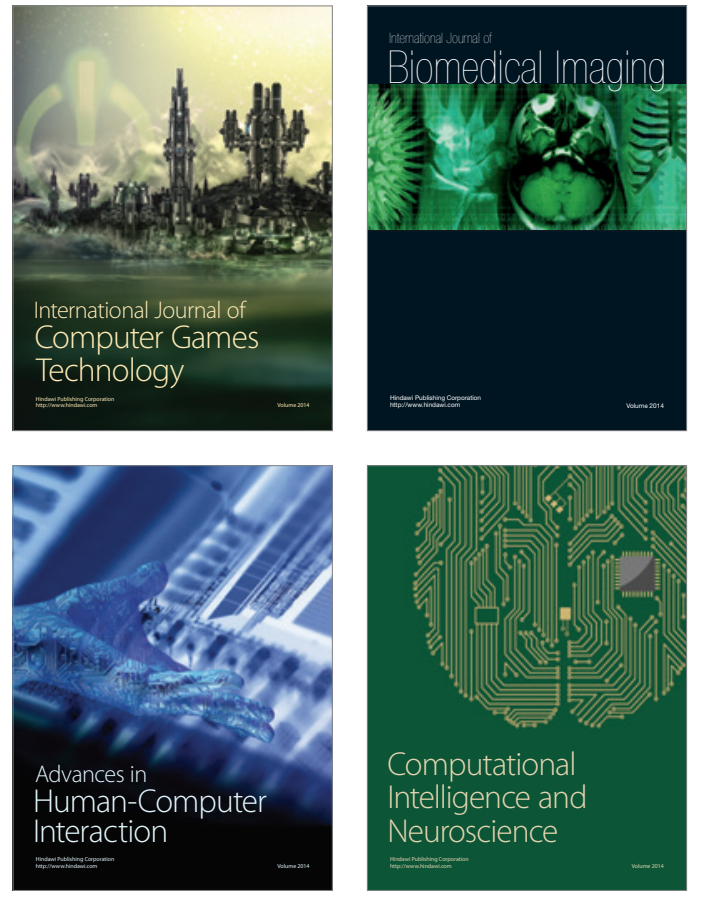
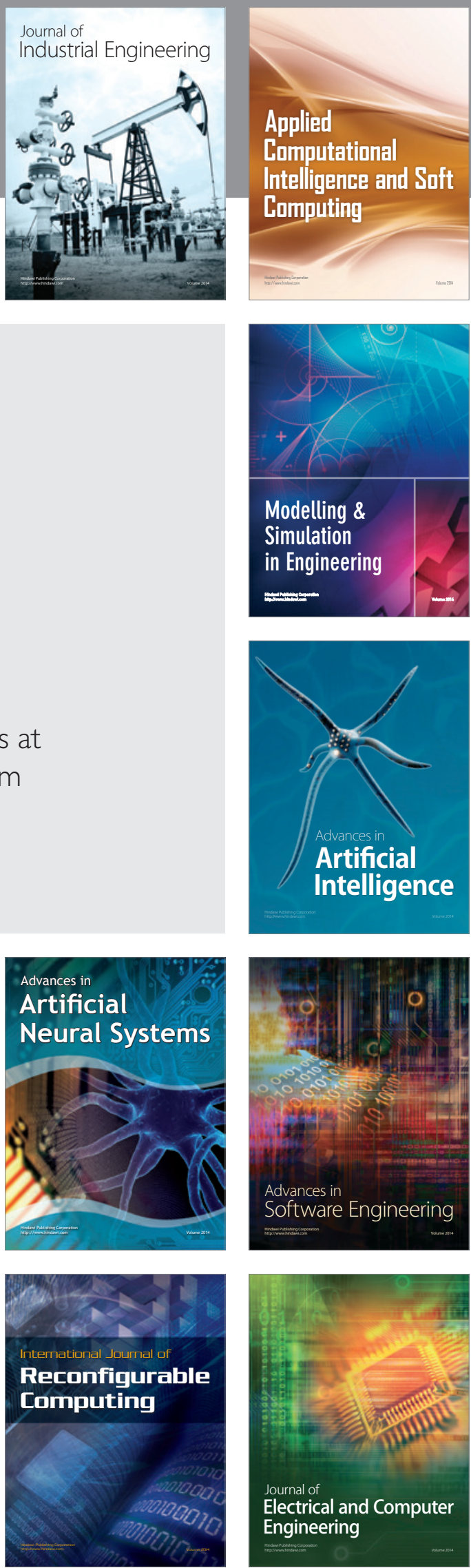